\title{
A Learning Analytics Methodology to Profile Students Behavior and Explore Interactions with a Digital Electronics Simulator
}

\author{
Mehrnoosh Vahdat ${ }^{1,3}$, Luca Oneto ${ }^{1}$, Alessandro Ghio ${ }^{1}$, Giuliano Donzellini ${ }^{1}$, \\ Davide Anguita ${ }^{2}$, Mathias Funk ${ }^{3}$, and Matthias Rauterberg ${ }^{3}$ \\ 1 DITEN - Università degli Studi di Genova, Genoa I-16145, Italy \\ mehrnoosh.vahdat@edu.unige.it, \\ \{luca.oneto, alessandro.ghio, giuliano.donzellini\}@unige.it \\ 2 DIBRIS - Università degli Studi di Genova, Genoa I-16145, Italy \\ davide.anguita@unige.it \\ 3 Department of Industrial Design, Eindhoven University of Technology, 5612AZ \\ Eindhoven, The Netherlands \\ \{m.funk,g.w.m.rauterberg\}@tue.nl
}

\begin{abstract}
The automatic collection of data concerning the interaction between students and Technology Enhanced Learning (TEL) systems has become increasingly common. Such data availability has led to applications of Learning Analytics (LA) techniques, characterized by the capability of extracting non-trivial patterns from them. In this framework, we describe the methodology we are adopting to explore the way students learn the concepts of digital electronics by exploiting LA. In particular, a simulator named DEEDS (Digital Electronics Education and Design Suite) is used, and we aim at extracting non-trivial knowledge from data, gathered through a logging application, properly realized for monitoring DEEDS usage $I^{1}$
\end{abstract}

Keywords: Learning Analytics, Technology Enhanced Learning, Simulator, Student Interaction.

\section{Learning Analytics with DEEDS Simulator}

Learning Analytics (LA) has developed remarkably in the last decade in the field of higher education by focusing on learning processes [1 3]. By adopting methods of analytics in e-learning, researchers gain more insight into the data collected from students through building models [4]. In the context of TEL, simulation-based environments are efficient tools to improve learning [5]. In this framework, we concentrate on higher education and use DEEDS simulator (Digital Electronics Education and Design Suite) [6] which is used for e-learning in

\footnotetext{
1 This work is supported partly by the Erasmus Mundus Joint Doctorate in Interactive and Cognitive Environments, funded by the EACEA Agency of the European Commission.
} 
digital electronics. We aim to extract precious knowledge via LA methods from data, gathered through a logging application called 'LA Data Collector' (LADC). This study was carried out on the students of Digital Electronics course at the University of Genoa during the spring semester, 2014.

In particular, a comprehensive experiment is designed and around 120 students participated in. An instructional design of the experiment to log the interaction data for comparing to the learning outcomes is performed. This leads us to discover common patterns and cross-correlations between variables for profiling students learning activities and attitudes. The activity data from the whole course by LADC is collected from over $95 \%$ of students (see Figure 1). During the experiment, observation, interview, and demographic questionnaire were carried out to acquire the learners' background, motivation, and feedback. A dataset concerning the learners' behavior and their key performance indicators, while interacting with the simulator is being prepared and will be made available as a valuable source for the research community.

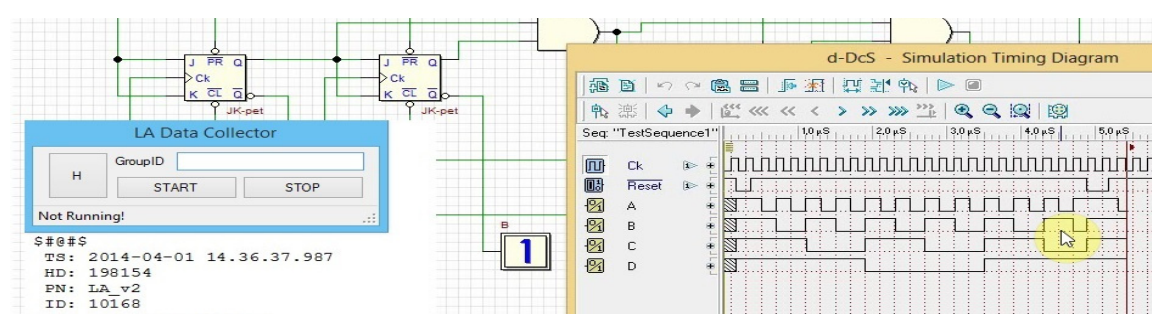

Fig. 1. DEEDS simulator used by a student trying to solve an exercise. On the lower left, the log data is shown as well as the LADC interface.

\section{References}

1. Brown, M.: Learning analytics: moving from concept to practice. Educause Learning Initiative (2012)

2. Shum, S.: Learning analytics. UNESCO Institute for Information Technologies in Education. Policy Brief (2012)

3. Bienkowski, M., Feng, M., Means, B.: Enhancing teaching and learning through educational data mining and learning analytics: An issue brief. US Department of Education, Office of Educational Technology, 1-57 (2012)

4. Siemens, G., de Baker, R.S.: Learning analytics and educational data mining: towards communication and collaboration. In: Proceedings of the 2nd International Conference on Learning Analytics and Knowledge, pp. 252-254. ACM (2012)

5. Donzellini, G., Ponta, D.: A simulation environment for e-learning in digital design. IEEE Transactions on Industrial Electronics 54(6), 3078-3085 (2007)

6. Ponta, D., Anguita, D., Da Bormida, G., Donzellini, G.: Ten years of activity on computer-aided learning for electronics: Needs, experiences, field evaluation, invited paper by taee 98, iii congreso sobre tecnologías aplicadas a la enseñanza de la electrónica. Universidad Politécnica de Madrid, Madrid, 16-18 (1998) 